\title{
Validity of Spontaneous Braking and Lane Changing with Scope of Awareness by Using Measured Traffic Flow
}

\author{
Kohei Arai \\ Graduate School of Science and Engineering \\ Saga University \\ Saga, Japan
}

\author{
Steven Ray Sentinuwo \\ Department of Electrical Engineering \\ Sam Ratulangi University \\ Manado, Indonesia
}

\begin{abstract}
This paper presents the validation method and its evaluation of the spontaneous braking and lane changing with scope awareness parameter. By using the real traffic flow data, the traffic cellular automaton model that accommodate these two driver behaviors, e.g., spontaneous braking and driver scope awareness has been compared and evaluated. The real traffic flow data have been observed via video-recording captured from real traffic situation. The validation results shown that by accommodate spontaneous braking and scope awareness parameters, the model can produced traffic flow's accuracy value 83.9\% compared to the real traffic flow data.
\end{abstract}

Keywords-traffic model validation; spontaneous braking; scope awareness; traffic cellular automaton.

\section{INTRODUCTION}

Validation is one of the important processes in the field of simulation and modeling. The validation process is concerned with determining whether the conceptual simulation model is an accurate representation of the system under study. However, the validation process cannot be defined to result a perfect model, since the perfect one would be the real system itself [1]. Naturally, any model is the simplification of the real world. On the other hand, simulation uses a model to develop conclusion providing insight on the behavior of the real world elements being studied. In the field of computer simulation, this term enhanced as the uses of computer programming to capture the real world situation. The origin of computer simulation and modeling is in the desire to forecast future behavior due to current phenomena. In the discipline of traffic engineering and transportation planning, computer simulation and modeling is needed because it can study models of traffic and its phenomena for analytical or numerical treatment can be used for experimental studies to describe detail evolution of the system over time, and produce the picture of current reality, as well as future estimation.

On the other words, the increasing trend of traffic congestion in most cities becomes the important issue in transportation system. Since travel demand increases at a rate often greater than the addition of road capacity, the situation will continue to deteriorate unless better traffic management strategies are implemented. To coup this problem, traffic simulation models are becoming as one of the important tool for traffic control. These simulation models is needed to asses, generate scenarios, optimize control, and estimate the future behavior of the system at the operational level. Through simulation the overall picture of traffic system can be pictured as well as the ability to assess current problems and the possible solutions immediately. Simulation and model can be a good tool to show some characteristics of complex traffic system, e.g., stable and unstable states, deterministic, chaotic or even stochastic behavior with phase transitions, fractal dimension and self-organized criticality. However, since the advance of technologies and application of transportation system in urban network and road way were not envisioned when many simulation models were developed, the existing models may not be directly applicable to such of this road system[2].

This paper is a continuation of the research work that have been done before[3]. The results from the previous research work have been evaluated by using real traffic data. In previous works, a simple traffic cellular automaton model has been developed in order to capture the real traffic flow with more naturally. The parameter of spontaneous braking and driver scope awareness was introduced. The spontaneous-braking probability rule captures the natural of braking behavior due to driver characteristic. The simulation results showed that traffic congestion can be effected by spontaneous braking behavior in the urban roadway with density $\rho \leq 0.75$. In the density $\rho>$ 0.75 , the effect of spontaneous braking in traffic congestion cannot be clearly distinguished. It is because in the high density level $\rho>0.75$ the congestion already occurred before spontaneous braking parameter applied.

Moreover, in addition to spontaneous braking parameter, the evaluation study of lane changing maneuvers has been done. The driver scope awareness parameter was introduced to reflect the visibility required by the driver to make a perception of a road condition and the speed of vehicle that exist within the certain area of the road before making a lane changing maneuver. Various value of scope awareness has been evaluated regarding their effect to the traffic flow. By taking into consideration the scope awareness parameter, the proposed traffic cellular automata model can reflect certain characteristics of lane changing maneuver in the real traffic situation.

This paper evaluates the simulation model that accommodates the driver behavior rules of spontaneous braking probability and lane changing scope awareness, by 
compare their simulation results to the real traffic data. The real traffic data has been observed via recorded traffic video.

This paper is organized as follow. The brief description about spontaneous braking and driver scope awareness in the lane changing maneuvers is quick reviewed in Section 2. Section 3 presents the validation and data gathering method that was used in this research. The comparison results and analysis are described in Section 4. Finally, in section 5, we present a summary and conclusion of this work

\section{SPONTANEOUS BRAKING AND SCOPE OF AWARENESS}

Spontaneous braking and scope awareness parameter introduced to reflect the characteristic of driver in real traffic situation. In reality, vehicle would make a braking as the response to avoid collision with another vehicle or avoid some obstacle like potholes, snow, or pedestrian that crosses the road unexpectedly. In many cases, the reckless driving behaviors such as sudden-stop by public-buses, motorcycle which changing lane too quickly, or tailgating make the probability of braking getting increase. Arai et.al.[3], using traffic cellular automaton (TCA) model to describe these characters through a traffic simulation and models. In that simulation, the effect of spontaneous braking and lane changing maneuvers on the traffic flow have been investigated. A set of movement rules include the parameter of spontaneous braking behavior can be described as follow:

- $\mathrm{R}(1)$ Acceleration:

$$
v_{(i)} \rightarrow \min \left(v_{(i)}+1, v_{\max }\right)
$$

- $\mathrm{R}(2)$ Deceleration

$$
v_{(i)} \rightarrow \min \left(v_{(i)}, \operatorname{gap}(i)\right)
$$

- $\mathrm{R}(3)$ Stochastic randomization

$$
p: v_{(i)} \rightarrow v_{(i)}-1
$$

- $\mathrm{R}(4)$ Spontaneous braking probability

$$
p_{b r}: v_{(i)} \rightarrow v_{(i)}-b
$$

- $\mathrm{R}(5)$ Driving

$$
x_{(i)} \rightarrow x_{(i)}+v_{(i)}
$$

Furthermore, in our previous research works, the effect of drivers' visibility and their perception (e.g., to estimate the speed and arrival time of another vehicle) on the lane changing maneuver have been investigated. The term of scope awareness was used to describe the visibility required by the driver to make a perception about road-lane condition and the speed of vehicle that exist in such road-lane. In that simulation study, several different values of scope awareness were examined to capture its effect on the traffic flow. A simple traffic cellular automaton model introduced to accommodate various braking characters of driver. Simulation results showed the ability of this model to capture the important features of lane changing maneuver and revealed the appearance of the short-thin solid line jam and the wide solid line jam in the traffic flow as the consequences of lane changing maneuver. The driver scope awareness rules can be summarized as follow:

$$
\begin{gathered}
\operatorname{gap}_{\text {same }}<v_{\text {current }} \\
\text { cell }_{\text {next }}=0 \\
\text { rand }()<p_{\text {change }} \\
\text { gap }_{\text {target }}>\text { gap }_{\text {same }} \\
\text { vehicle } \text { back }_{\text {gapack }} ; X\left(\text { vehicle }_{\text {back }}\right) \in S a
\end{gathered}
$$

\section{VALIDATION METHOD}

Validation is used to determine the real world system being studied is accurately represented by the simulation model. Referring to ISO standard, the following steps in validation are listed[4]:

- Component testing: checking of software subcomponent (the model);

- Functional validation: checking of model capabilities and inherent assumptions;

- Qualitative verification: comparison of predicted traffic behavior with informed expectations.

- Quantitative verification: comparison of model predictions with reliable experimental data.

The first two of these items are usually based on simple test cases and do not require empirical data. The third is based on comparison with observation, and the last on comparison with quantitative and experiment data.

Often, the test has to be done in several times to obtain the best result of validity. By a thorough analysis of the simulation's output data then the best result would be taken. If the model's output data closely represents the expected values for the system's real-world data, then validity is more likely.

When a model has been developed for an existing system, a validity test becomes a statistical comparison. Data collected from the situation of real system can be used as theoretical comparator[5]. However, when the system does not yet exist, validity becomes harder to prove. In many cases, validity cannot be definitely proven until some point in the future when the system being modeled has been deployed and running.

\section{A. Actual Data Gathering}

Empirical data is used in validation of simulation results. The data used in this validation is based on video analysis. The usual approach towards data recording is observation and counting. The analysis is done manually, i.e., there was no automatic device that extracted the information from video. The evaluation of the data presented below is based on the following assumptions and methods. Since we interest to evaluate the effect of spontaneous braking behavior in the 
traffic flow then their number through video recording have been counted.

A field observation using micro scenario where the actual traffic condition were captured by a 30 minutes video recorded. The parameter that was counted is traffic flow and the number of spontaneous braking. The actual data were taken from twolane urban roadway with a length approximately $500 \mathrm{~m}$. The video camera was placed on the pedestrian bridge. Figureure 1 shows the observation location. Among the location, there are two traffic signal at the end of lane, then to distinguish between the normal braking and the spontaneous braking of vehicle, we use an assumption. For the vehicle that stop due to traffic signal would be categorized as normal braking, other than that would be categorized as spontaneous braking.

Through the video recording, the number of traffic flow and spontaneous braking has been counted for each of the recorded video data. The observation data were taken from 16 different real urban road traffic video data. These data were taken in the morning and afternoon as being assumed as peak traffic time. Table 1 shows the average density values of the observation results. In traffic data analysis, there are three related types of data: speed, flow, and density. Speed $v(\mathrm{~km} / \mathrm{hr})$ is defined as the distance covered per unit time.

Since the speed of every vehicle is almost impossible to track on the roadway, therefore, in practice, average speed is based on the sampling of vehicles over a period of time or area and is calculated and used in formulae. Some sensing systems can directly measure it. Flow $q$ is the rate in which vehicles arrive at a particular point on a roadway and described in terms of vehicles per hour (cars/hr). Traffic sensing systems usually record the traffic volume, which is the actual number of vehicles to arrive during a sampling period (e.g., 30 seconds). Thus, volume can be converted to a flow rate by multiplying the recorded volume by the number of sampling periods in an hour. Density $k$ is defined as the number of vehicles per unit area of the roadway. The density value is described in terms of vehicle per unit area (cars $/ \mathrm{km}$ ). By measure flow and speed, the density is calculated by dividing the flow rate by the speed.

Since the real traffic data was recorded in 30 minutes video then based on this traffic flow counter, then we estimated the traffic flow for 1 hour. The average speed of vehicles was obtained by field experiment, e.g., driving a car along the observation area then calculated the average speed among such area. Once the flow $q$ and speed values $v$ were obtained then the density value $k$ in the observation area was calculated by using the equation:

$$
k=\frac{q(\operatorname{cars} / h r)}{v(k m / h r)}
$$

In the traffic cellular automaton model, most of the typical models use a consideration that one cell of the simulation model equal to the $7.5 \mathrm{~m}$ length of the real system. This value is considered as the length of vehicle plus the distance between vehicles in a stopped position. Referring to this assumption then for $1 \mathrm{~km}$ road length there must be 133 vehicles that equal to maximum density among the road lane. Table 1 shows the summarized data. Data from the sequences video that have same density value have been calculated and retrieved their average value.

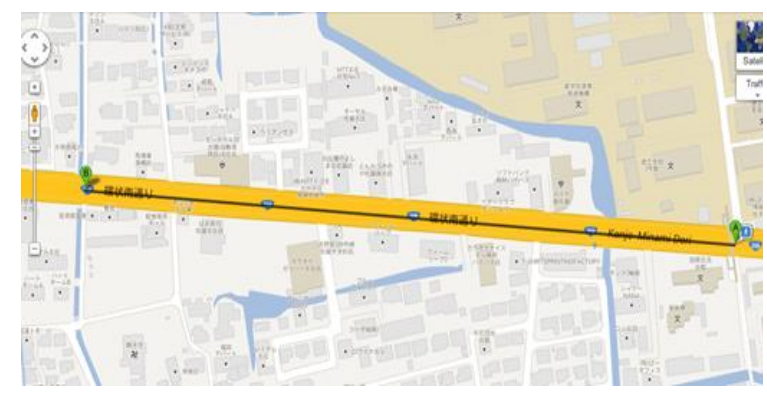

Fig.1. The location of observation.

TABLE I. THE AVERAGE VALUES OF THE OBSERVATION RESUlts

\begin{tabular}{|c|c|c|c|c|}
\hline $\begin{array}{c}\text { AVG. } \\
\text { SPEED } \\
\mathbf{( k m / h r )}\end{array}$ & $\begin{array}{c}\text { DENSITY } \\
\text { (cars/km) }\end{array}$ & $\begin{array}{c}\text { DENSITY } \\
(\% / \mathbf{k m})\end{array}$ & $\begin{array}{c}\text { SP. } \\
\text { Braking } \\
\mathbf{( \% )}\end{array}$ & $\begin{array}{c}\text { Real Data } \\
\text { FLOW } \\
\text { (cars/hr) }\end{array}$ \\
\hline \hline 50 & 18.28 & 7 & 4 & 914 \\
\hline 50 & 20.18 & 8 & 4 & 1009 \\
\hline 40 & 24.3 & 9 & 4 & 972 \\
\hline 40 & 25.8 & 10 & 5 & 1032 \\
\hline 40 & 28.75 & 11 & 3 & 1150 \\
\hline
\end{tabular}

\section{COMPARISON AND ANALYSIS}

In this observation, we evaluated the number of traffic parameters provided by video recording, i.e., spontaneous braking events, flow number, cars density value, and average speed of the cars. There were several types of vehicle exist in this observation, e.g., motorbike, bus, passenger car, and bicycle. However, in the data analyzing, we just considered for the car types, include truck and bus. After those traffic parameters had been calculated then through the simulation model those real traffic data has been compared. We compared the number of traffic flow resulting from real traffic data and the proposed model. This evaluation used spontaneous braking number and density level from real traffic data. By using those parameters as input value, we obtained the traffic flow results of simulation model.

The comparison result of traffic flow is shown by Figure. 2. Referring to the video observation, in the simulation model we used the probability of lane changing 0.1 . In this evaluation, we also compared the traffic flow result by using NagelSchreckenberg model[6]. The comparison result shows the model that accommodates spontaneous braking and driver scope awareness produced the better result of traffic flow rather than the original Nagel-Schreckenberg model[6]. The accuracy values between real data flow and our model are presented in Table II, as well as Nagel-Schreckenberg model. It can be seen from the comparison results (Figure. 2), there is a discrepancy between real data and simulation results. The simulation model produced .25 higher cars flow than real traffic flow. Therefore, in this work, we also tried to compare the real traffic data flow to various values of spontaneous braking probability and scope 
awareness. Figure 3 presents the scatter graph of comparison between real data flow and several spontaneous braking probability values. Meanwhile, Figure. 4 presents the comparison result between real traffic flow data and several value of driver scope awareness parameter, i.e., scope awareness 3 cells and scope awareness 6 cells, respectively.

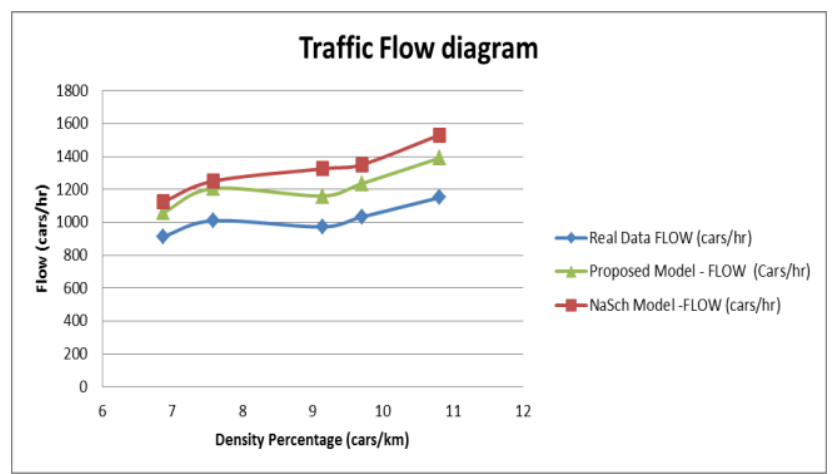

Fig.2. Comparison results of real data vs proposed model vs NaSch model.

TABLE II. TRAFFIC Flow ACURRATION VALUES

\begin{tabular}{|l|c|}
\hline \multicolumn{1}{|c|}{ Data Source } & $\begin{array}{c}\text { Accuration } \\
\text { (\%) }\end{array}$ \\
\hline \hline Real Data & 100 \\
\hline NaSch Model & 75.9 \\
\hline Proposed Model & 83.9 \\
\hline
\end{tabular}

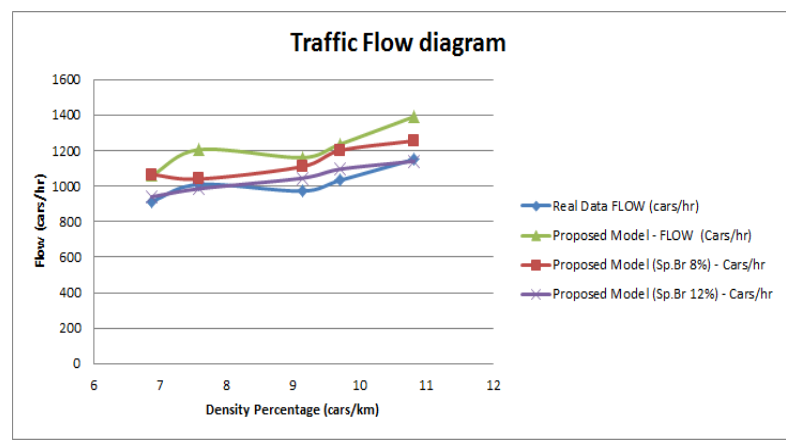

Fig.3. Comparison of real data to several spontaneous braking values

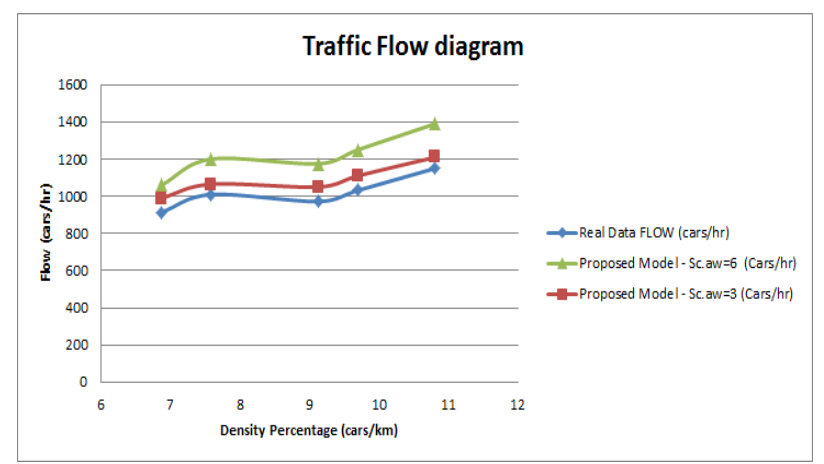

Fig.4. Comparison of real data to several Scope Awareness values

\section{A. Traffic Flow and Average Speed Estimation}

In addition, this paper also evaluated and made estimation for traffic flow and average speed on every density values. Referring to the Figure 3, it can be seen that by using spontaneous braking probability value 0.12 , the simulation can produces a similar flow behavior to the real traffic flow data.

Thus, in order to predict the future traffic behavior, the simulation model used spontaneous braking probability 0.12 . Figure 5 and Figure 6 present the traffic flow and speed estimation for all density values, respectively. The horizontal axis represents the percentage of car's density levels on the roadway-length while vertical axis in Figure 6 represents the percentage of the cars that can move at one time step in simulation. In Figure 7, the vertical axis describes the maximum speed that can be reached by the car at the specific density level.

\section{CONCLUSION}

A validation of spontaneous braking and scope awareness model using measured real traffic flow has been introduced. This validation shows that the traffic cellular automaton model that accommodate the probability of spontaneous braking and scope awareness have given more accurate description about traffic flow situation. Moreover, this paper also presents the estimation behavior of the traffic flow and average speed on every density values.

\section{REFERENCES}

[1] J. P. C. Kneijnen, "Theory and Methodology Verification and validation of simulation models," European Journal of Operational Research, vol. 82, pp. 145-162, 1995.

[2] S. Boxill and L. Yu, "An Evaluation of Traffic Simulation Models for Supporting ITS," Houston, 2000

[3] K. Arai and S. Sentinuwo, "Spontaneous-braking and lane-changing effect on traffic congestion using cellular automata model applied to the two-lane traffic," International Journal of Advanced Computer Science and Applications, vol. 3, no. 8, pp. 39-47, 2012.

[4] H. Klüpfel, "A cellular automaton model for crowd movement and egress simulation," Universitat Duisburg-Essen, 2003.

[5] R. McHaney, Understanding computer simulation. RogerMcHaney \& Ventus Publishing Aps, 2009.

[6] K. Nagel and M. Schreckenberg, "A cellular automaton model for freeway traffic," Journal of Physics I France, vol. 2, no. 12, pp. 22212229, 1992.

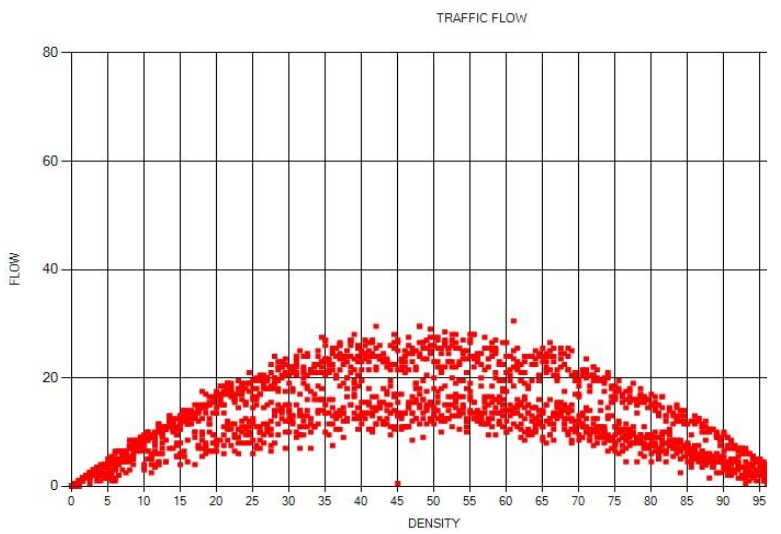

Fig.5. Traffic flow estimation for all density values 
AVERAGE SPEED

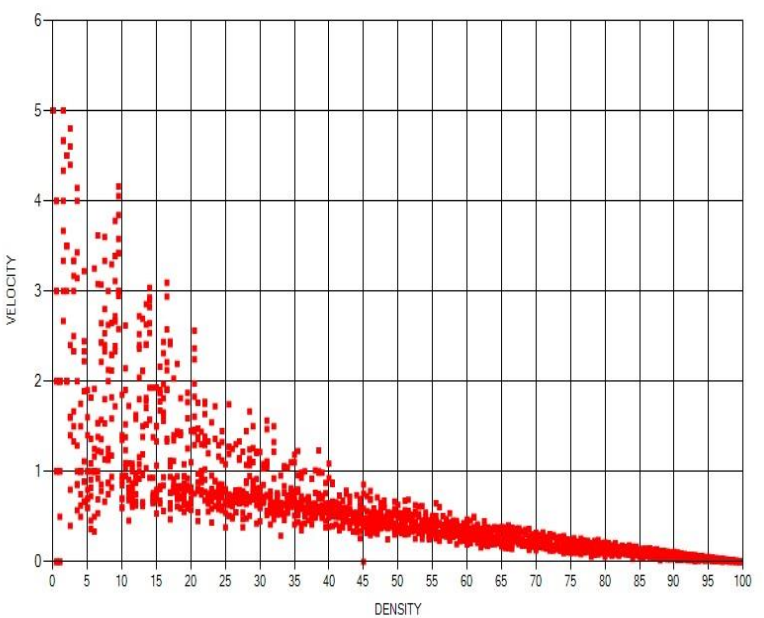

Fig.6. Average speed estimation for all density values

\section{AUTHORS PROFILE}

Kohei Arai received BS, MS and PhD degrees in 1972, 1974 and 1982 respectively. He was with The Institute for Industrial Science and Technology of the University of Tokyo from April 1974 to December 1978 and also was with National Space Development Agency of Japan from January, 1979 to March, 1990. During from 1985 to 1987, he was with Canada Centre for Remote Sensing as a Post-Doctoral Fellow of National Science and Engineering Research Council of Canada. He moved to Saga University as a Professor in Department of Information Science on April 1990. He was a counselor for the Aeronautics and Space related to the Technology Committee of the Ministry of Science and Technology during from 1998 to 2000. He was a councilor of Saga University for 2002 and 2003. He also was an executive councilor for the Remote Sensing Society of Japan for 2003 to 2005. He is an Adjunct Professor of University of Arizona, USA since 1998. He also is Vice Chairman of the Commission A of ICSU/COSPAR since 2008. He wrote 30 books and published 322 journal papers

Steven Ray Sentinuwo, received the B.Eng. degree in Electrical Engineering from Sam Ratulangi University, and the M.Eng. degree in Information Engineering, from University of Indonesia, in 2004 and 2006, respectively. $\mathrm{He}$ is currently a $\mathrm{PhD}$ Student at Information Science in Saga University, Japan. His research interest includes robot path planning, traffic simulation and modeling, and information system management. 